Acta Crystallographica Section D

Biological Crystallography

ISSN 0907-4449

\section{Eric Thouvenin and Elizabeth Hewat*}

Institut de Biologie Structurale J.-P. Ebel, 42 Rue Jules Horowitz, 38027 Grenoble, France

Correspondence e-mail: hewat@ibs.fr

\title{
When two into one won't go: fitting in the presence of steric hindrance and partial occupancy
}

Combining structural data from cryo-electron microscopy (cryo-EM) and X-ray crystallography to give pseudo-atomic models of large molecular complexes has proved particularly suitable for studying viruses and viral complexes. Several groups are developing programs to fit $\mathrm{X}$-ray data to EM data. These programs are in general tailored to particular problems with regard to size, symmetry, number of rigid bodies, resolution etc. Here, two approaches are described to fitting $\mathrm{X}$-ray data to EM data in the presence of steric interference and their relative merits and limitations are indicated. These fitting techniques are applied to the case of the rotavirus double-layered particle (DLP) in complex with antibodies which inhibit the transcription of mRNA by the DLP. This is a particularly good test case, as the cryo-electron microscopy map of the DLP-Fab complex, the X-ray structure of the viral protein (VP6) and also that of the VP6-Fab complex are available. The estimation of partial occupancy is also considered.

\section{Introduction: virus-antibody complexes}

The recognition of an intruding virus particle by an antibody is an event that alerts the organism's immune-response system for action. The defence role played by antibodies alone and in concert with other elements of the body's immune system is however not entirely understood. The in vitro study of monoclonal antibodies (MAb) which neutralize viruses show that several different neutralization mechanisms exist and that any one MAb may invoke a subtle combination of these mechanisms (Mosser et al., 1989). Understanding how antibodies neutralize a virus at the atomic level is a major challenge in structural virology. Antibodies bind to viruses forming large molecular complexes which are both flexible and fleeting and their structure is therefore not easily amenable to solution by X-ray crystallography. The developing methodology of combining structural data from cryo-electron microscopy and X-ray crystallography to give a pseudo-atomic model is proving highly appropriate in this context (Wang et al., 1992; Porta et al., 1994; Baker \& Johnson, 1996). While neutralizing MAbs are of particular interest (Smith, Olson, Cheng, Liu et al., 1993; Smith, Olson, Cheng, Chase et al., 1993; Hewat et al., 1997, 1998; Verdaguer et al., 1999), antibodies which simply mark a given viral protein (Trus et al., 1992) or which modify the viral activity (e.g. inhibit mRNA transcription; Lawton et al., 1999) also merit structural study. The structure of virus-antibody complexes provides information not only on how antibodies neutralize viruses but also on how antibodies interact with antigens in general, in particular the flexibility of antibodies and the distance between two binding
Received 21 March 2000 Accepted 28 June 2000 
sites of one antibody (Smith, Olson, Cheng, Chase et al., 1993; Hewat \& Blaas, 1996).

The cryo-EM map of the virus-Fab complex alone gives information about the relative positions and orientation of the virus and Fabs, the Fab (or MAb) flexibility, the occupancy of the Fab sites and the distance between binding sites. The pseudo-atomic structure derived from the combined data goes further by revealing whether there is any major change in the

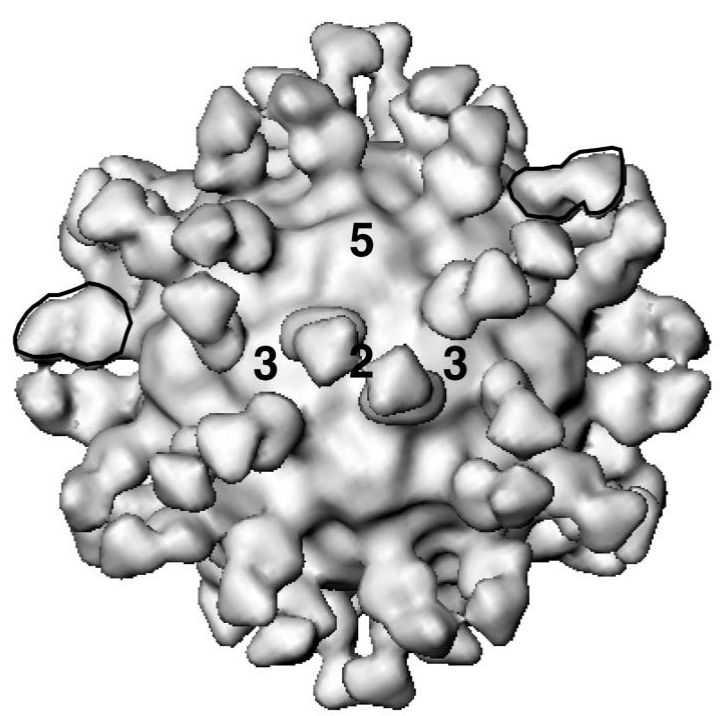

(a)

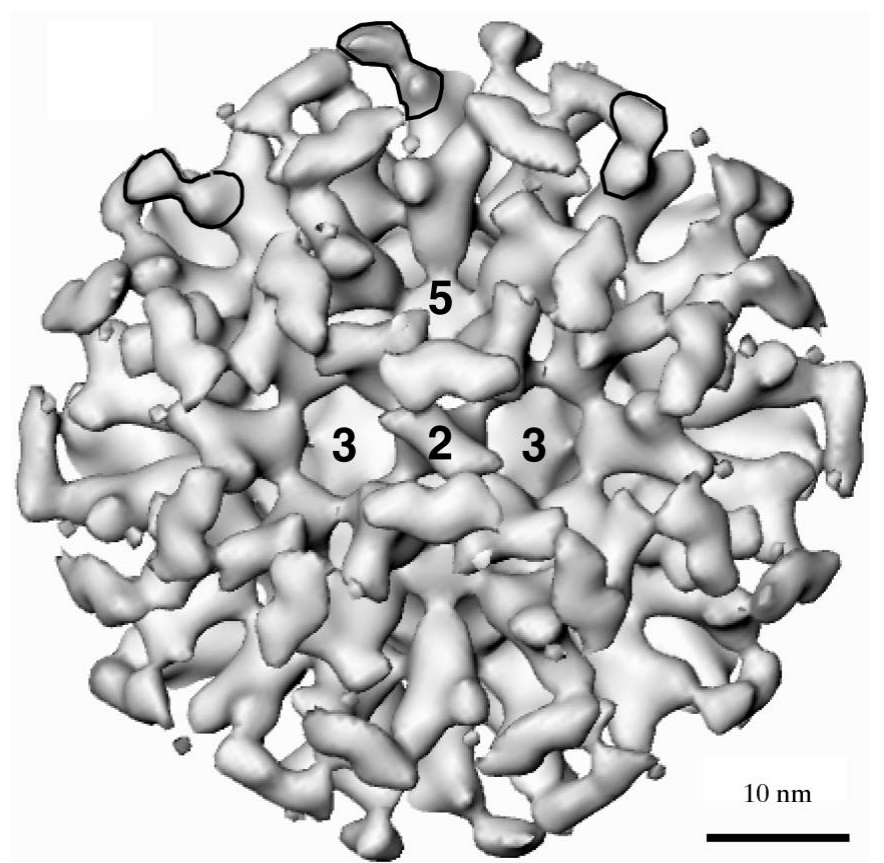

(b)

Figure 1

Isosurface representations of the cryo-EM maps of $(a)$ the complex HRV2-MAb-8F5 and (b) the complex RHDV-MAb-E3. In both of these complexes the whole IgG has a bivalent attachment to the virion but the $\mathrm{Fc}$ fragment is not visible in the reconstructions. This is attributed to movement of the Fc fragment. Several individual Fab fragments are outlined in $(a)$ and $(b)$. The Fabs in $(b)$ appear smaller because their occupancy is only about $50 \%$. Both representations are viewed down the twofold axis and the icosahedral axes are marked 2, 3 and 5. viral capsid on binding of the Fab, whether the cell-receptor site on the viral capsid is obscured and confirming whether the antibody binding is monovalent or bivalent. The magnitude and extent of the density attributed to the antibody give some indication of its occupancy and movement (or flexibility). In every reconstruction where a whole $\mathrm{IgG}$ is attached to an icosahedral virus, the $\mathrm{Fc}$ fragment is either not visible or only very weakly represented. This is taken to indicate movement of the $\mathrm{Fc}$ fragment associated with the highly flexible nature of the antibody hinge region (Figs. $1 a$ and $1 b$ ).

Since many spherical viruses have icosahedral symmetry, steric hindrance between the Fabs on neighbouring epitopes can occur, with consequent partial occupancy. This complicates the interpretation of the density map (Fig. 1b). The Fab occupancy will reflect the underlying differences in the conformation and accessibility of the viral proteins in different symmetry environments. This is a factor which can influence the neutralizing capacity of the antibody, as not all possible epitopes can be covered. Four of the eight virus-antibody complexes studied in our laboratory exhibited steric hindrance between neighbouring Fabs. Thus, steric hindrance is not a particularly rare occurrence in virus-antibody interactions. In this paper, we aim to address the aspects of the fitting of X-ray to EM data in the presence of steric hindrance and partial occupancy. Other more general aspects of X-ray to EM fitting will be presented in other papers in this issue. We will describe briefly the case of rabbit haemorrhagic disease virus (RHDV) in complex with a neutralizing antibody and outline the reasoning which led to our interpretation of the cryo-EM density map (Thouvenin et al., 1997). The X-ray to EM fit in this case was visual (i.e. qualitative). We then present a more detailed description of the fitting techniques adopted for the case of the rotavirus double-layered particle (DLP) in complex with antibodies which inhibit the transcription of mRNA by the DLP (Thouvenin et al., in preparation). This is a particularly good test case, as we already possess the X-ray structure of the viral protein (VP6) and also of the VP6-Fab complex. The estimation of occupancies will also be considered. There are currently many different groups developing EM to X-ray fit programs. Not surprisingly, they are tailored to particular problems with regard to size, symmetry, number of rigid bodies, resolution etc. Here, we endeavour to describe two approaches to the fitting in the presence of steric interference and indicate their relative merits and limitations.

\section{Fitting X-ray structures to cryo-EM maps of virus- antibody complexes with no steric problems}

Fitting X-ray structures to cryo-EM maps of virus-antibody complexes is in many cases one of the more tractable fitting problems and can easily be performed in real or reciprocal space (Hewat et al., 1997; Che et al., 1998). The characteristic shape of a Fab fragment facilitates the task, so that even at low resolution a fit is possible, as the binding site (paratope) of the Fab is known. It will be noted, however, that there may be a certain ambiguity in the fit arising from the presence of a pseudo-twofold symmetry axis coincident with the long axis of 
the Fab. This ambiguity can be resolved if the elbow angle of the Fab is large, but additional information is often useful in confirming the fit or resolving the ambiguity. For example, when the structure of the Fab co-crystallized with a peptide that corresponds to the viral epitope is known, the position of the peptide must be compatible with the position of the epitope on the viral surface. An illustration of this is found in case of human rhinovirus serotype 2 (HRV2) complexed with the neutralizing MAb-8F5 (Hewat \& Blaas, 1996). Here, the structure of the Fab fragment co-crystallized with the 15-mer peptide which mimics the contiguous epitope on the viral capsid is known (Tormo et al., 1994). The difference of $1 \%$ in the cross correlation coefficient between the best fit Fab position and the $180^{\circ}$ rotated position at a resolution of $25 \AA$ proved to be meaningful. The position of the terminal residues of the 15-mer peptide could only be joined to the corresponding amino acids on the viral capsid in the best fit position. This also coincided with the best orientation given by observation of the inclination of the Fab elbow angle in both maps (Fig. 1a).

The current precision of the rigid-body fit of roughly $4 \AA$ at 20-30 A resolution was confirmed for a HRV14-Fab complex where the pseudo-atomic structure (Smith, Olseon, Cheng, Liu et al., 1993) and the atomic X-ray structure (Smith et al., 1996) of the complex were both determined. This precision is not quite sufficient to determine detailed amino-acid interactions, but it is certainly sufficient to predict useful pointmutation experiments. Nor can adaptive movements of interacting loops be determined, except in special cases as evoked above where a peptide which mimics the epitope is co-crystallized with the Fab.

\section{Preparation of the EM map for fitting the X-ray structure: 'CTF' correction}

In order to compare X-ray and EM maps, some form of normalization is required; in particular, the contrast-transfer function (CTF) modulation of the EM map must be corrected. A useful preparatory step is to calculate a low-resolution map of the components of the complex from the X-ray atomic structures. The hand of the EM map may then be determined by comparison of the viral capsid density in both maps, if this has not been performed by metal shadowing or tilting experiments. A more precise scale for the EM map may also be obtained by comparison with the X-ray map of the capsid, e.g. by cross correlation of the spherically averaged density of the capsid. Once a first visual fit of the virus and Fab is obtained using, for example, the program $O$ (Jones et al., 1991), a practical 'CTF' or more precisely an 'EM to X-ray correction' can be estimated. The EM CTF essentially modulates the amplitudes of the Fourier components as a function of radius and flips the phases at well defined radii determined by the defocus of the image. The CTF can be corrected quite well for the higher resolution data (Conway \& Steven, 1999) using the theoretical curves determined from the measured zeros of the CTF. However, for the lower resolution data up to the first maximum of the CTF, the correction is not easy to estimate theoretically. Also, the effective Debye-Waller temperature-factor correction to bring the cryo-EM map into line with the X-ray map is best estimated by direct comparison of the data sets. The more common procedure is to apply a large Debye-Waller correction (500-6793 $\AA^{2}$ ) to the X-ray data (Belnap et al., 1999), thus reducing the contribution of the higher resolution data in order to bring it into line with the EM data. We choose to bring the EM map into line with the X-ray map, thus increasing the contribution of the higher resolution data. Thus, we give more weight to the higher resolution data in the evaluation of the cross-correlation coefficient than is commonly the case.

The following practical method for making the 'CTF' correction has proved satisfactory in that it improves the EM to X-ray fit (Hewat et al., 1997) as estimated by the $R$ factor in $X$-PLOR. A map of the virus-Fab complex is created by visually fitting the $\mathrm{X}$-ray structure of the Fab and the virus to the EM map of the complex. The EM map is masked in order to exclude all density except that corresponding to the capsid and the Fab. This is achieved by subtracting the mean density from the EM map and setting all density below zero to zero, with the protein density positive. The density corresponding to the viral RNA is also set to zero. The Fourier transform of both maps (i.e. the masked EM map and the equivalent X-ray map) are compared and the ratio of the spherically averaged Fourier amplitude is smoothed to give a radial 'CTF' correction for the EM map (Fig. 2).

\section{Fitting X-ray structures into cryo-EM maps of virus- antibody complexes with steric interference}

Steric hindrance between Fabs, which gives rise to partial occupancy, results in cryo-EM maps with the viral capsid well represented by a high density but with overlapping Fabs represented by various lower densities. The map is an icosahedrally averaged map. Thus, an isodensity surface with a single threshold will not give an easily interpretable representation of the virus-antibody complex. Fitting the X-ray structure of the Fab fragments to the EM map is more complex.

\subsection{An example of a qualitative fit: rabbit haemorrhagic disease virus complexed with a strongly neutralizing MAb}

Rabbit haemorrhagic disease virus (RHDV), a member of the Caliciviridae family, is the causative agent of a highly contagious disease in wild and domestic rabbits, first described in China in 1984. It has been responsible for great economic losses in rabbit production. The virion is $40 \mathrm{~nm}$ in diameter and consists of 180 copies of a single $60 \mathrm{kDa}$ protein arranged as dimeric arches on a $T=3$ icosahedral lattice. The structure of a closely related primate calicivirus has been determined by cryo-electron microscopy (Prasad et al., 1994) and the structure of a human calicivirus, the Norwalk virus, has been determined by X-ray crystallography (Prasad et al., 1999). 
Baculovirus-expressed capsid protein self-assembles to form virus-like particles (VLPs).

In cryo-electron microscope images of the RHDV VLP complexed with a strongly neutralizing MAb-E3, the VLP appear to be well decorated with MAb (Thouvenin et al., 1997). Three-dimensional reconstructions of the complex show that the density in the Fab domains is only about half that in the capsid and hence the occupation of Fab sites is incomplete, while there is no density which can be attributed to the Fc fragment (Fig. 1b). The density near the VLP, which we attribute to the variable modules of the Fab, is roughly $50 \%$ of the capsid density, while the density further from the VLP, which we attribute to the constant domains, is less than $40 \%$ and is spread over a larger volume. As there is no appreciable aggregation of the complexes and considering the form of the density, we conclude that the MAbs have a bivalent attachment to the VLP. There are manifestly three epitopes on neighbouring arches, related by a local threefold

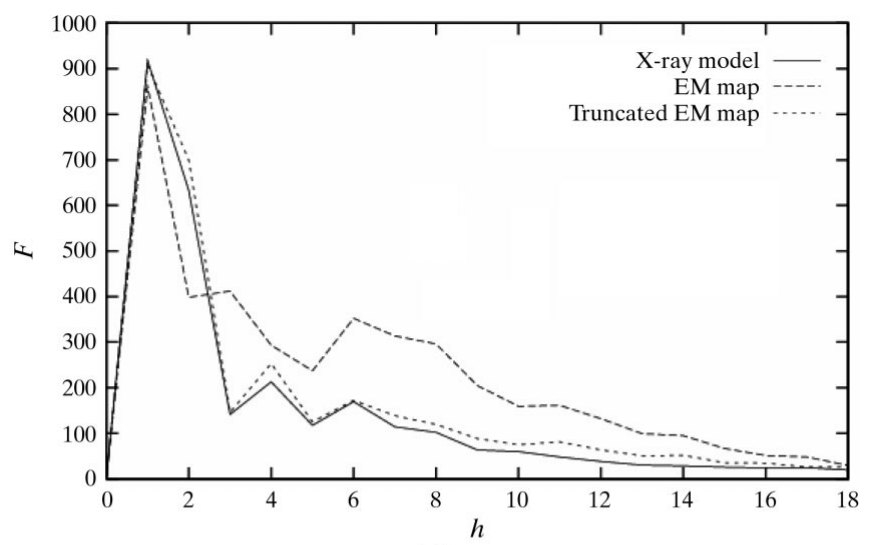

(a)

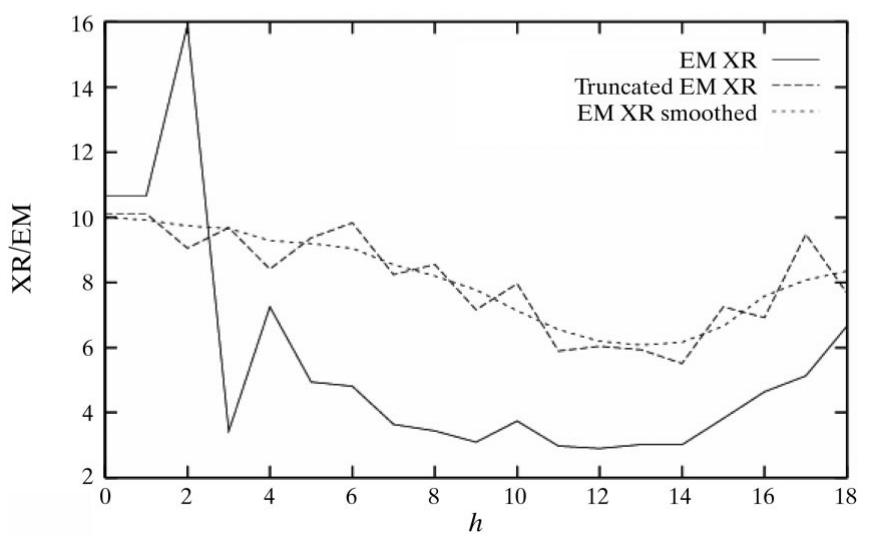

(b)

Figure 2

Curves for the estimation of the 'CTF' or 'EM to X-ray' correction. (a) Graph of spherically averaged Fourier amplitudes of the X-ray model (virus plus Fabs), the EM map and the truncated EM map (i.e. the EM map with the mean subtracted and all density below zero set to zero). The $h$ corresponds to a cubic unit cell of $454.4 \AA$. (b) Curves of the X-ray amplitude divided by the EM amplitude. The scale is arbitrary. Only the data from the truncated EM map gives a reasonable correction. The correction curve is smoothed visually before application to the EM amplitudes. These curves relate to the HRV2-MAb-8F5 complex. icosahedral symmetry axis, which compete for each MAb. Each MAb may be attached to any pair of the three epitopes. We were able to model this in the density with pairs of Fab representing each MAb. In this model, each dimeric arch has two epitopes such that the bound Fabs overlap slightly. This correlates with the observed occupancy of roughly $50 \%$ for each constant module. It is notable that the MAbs are bound to epitopes which are related by an approximate threefold symmetry axis rather than a twofold axis: this bears witness to the torsional flexibility of the hinge region of the MAb (Fig. 3).

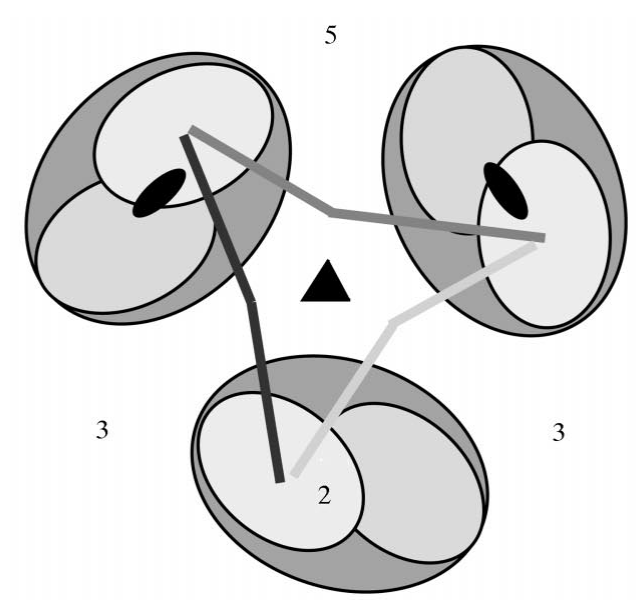

(a)

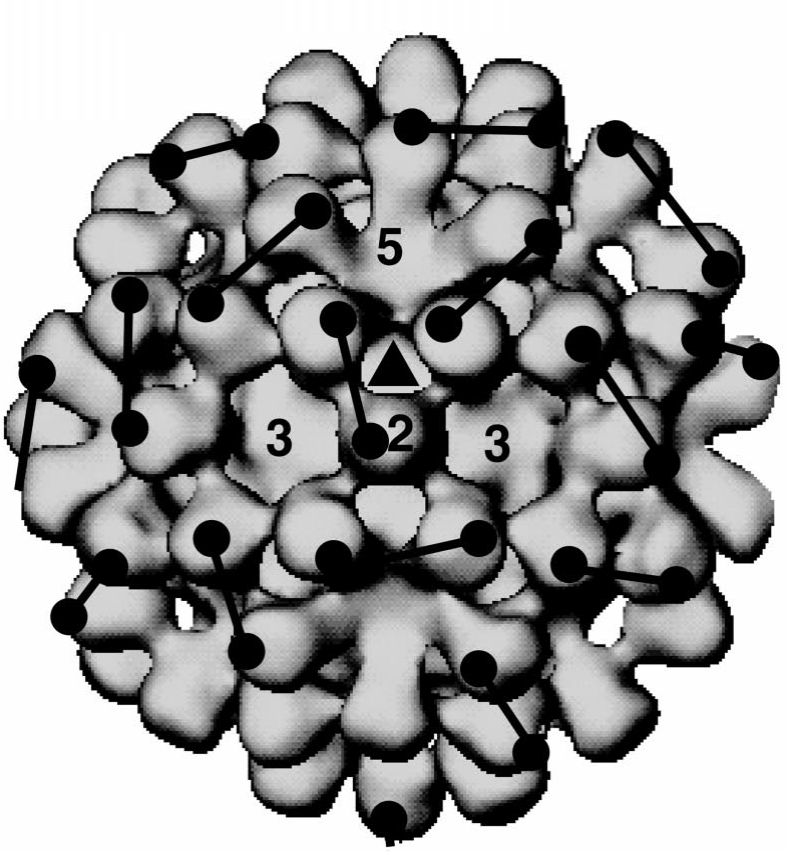

(b)

Figure 3

Schematic diagrams of the epitopes of the neutralizing MAb-E3 on the RHDV capsid. The icosahedral axes are marked 2, 3 and 5 and the local two- and threefold axes are marked with symbols. In $(a)$, three dimeric arches of the capsid are represented in dark grey and possible pairs of binding sites for one MAb are shown linked by lines in different shades of grey. The overlapping epitopes on each arch are indicated. A schematic diagram of one of the many possible distributions of mAb-E3 on the capsid is shown in $(b)$. (Adapted from Fig. 6 in Thouvenin et al., 1997.) 


\subsection{Quantitative fitting in the presence of steric hindrance}

The essential problem in fitting in the presence of steric hindrance is to allocate the density in the EM map to the constituent molecules as precisely as possible, since the fitting routines considered here are only capable of refining the fit given a first approximation. Particularly for fitting in real space, the removal of density corresponding to the viral capsid is a necessary step to prevent the Fab sinking slightly into the capsid density. This may be accomplished either by subtracting the EM density map of the capsid (difference imaging) or by masking the capsid density from the EM map with an atomic mask. All the operations described are performed using a combination of CCP4 (Collaborative Computational Project, Number 4, 1994) and in-house routines. We employ the CCP4 routines PDBSET to move PDB coordinates and define the extent of a molecule, $S F A L L$ to calculate structure factors from atomic coordinates followed by FFT to calculate the low-resolution density maps and NCSMASK to calculate molecular masks to be applied to EM and X-ray density maps. We use in-house programs to interpolate the rotated and translated density map and calculate cross-correlation coefficients immediately. This speeds up the fitting process by a factor of ten compared with the use of the two equivalent $C C P 4$ routines MAPROT and OVERLAPMAP.

Two methods have been tested for fitting overlapping Fabs. These are methods which maximize the cross-correlation coefficient in real space between the prepared EM map and the X-ray map filtered to the same resolution. Both require as input a first estimate of the Fab positions and occupancies. The first involves simulating the density maps of both of the overlapping Fabs from a first fit (visual fit) position and adding the maps together weighted to represent the relative occupancy (combination method). The contribution of one Fab is held constant while the second is scanned in position, orientation and occupancy in search of the maximum cross correlation. The role of each Fab is then alternated and the cycle repeated until convergence. The second is basically a difference technique which aims at extracting the density corresponding to each Fab separately (extraction method). The first estimate Fab positions (visual fit positions) are used to mask off each Fab separately and to estimate the overlapping region where the density is apportioned to each map in proportion to the estimated relative occupancy. The fit of one Fab is then refined in position, orientation and occupancy independently of the other Fab. The role of each Fab is then alternated and the cycle repeated until convergence (Fig. 4). The size of the mask must be roughly $5 \AA$ about each atom. The crosscorrelation coefficient employed is

$$
r=\frac{\sum_{j}\left(\rho_{a_{i}}-\bar{\rho}_{a}\right)\left(\rho_{b_{i}}-\bar{\rho}_{b}\right)}{\left[\sum_{j}\left(\rho_{a_{i}}-\bar{\rho}_{a}\right)^{2} \sum_{j}\left(\rho_{b_{i}}-\bar{\rho}_{b}\right)^{2}\right]^{1 / 2}},
$$

where $\bar{\rho}_{a}$ is the mean of the $\rho_{a}$ and $\bar{\rho}_{b}$ is the mean of the $\rho_{b}$.

\subsection{An example of a quantitative fit: two rotavirus double- layered particle-Fab complexes}

Rotaviruses, members of the Reoviridae family, are the major cause of acute gastroenteritis in children. They consist of a multi-shelled icosahedral capsid which encloses 11 ds-RNA molecules and two core proteins, VP1 and VP3. On entering the host cell, the virion loses its outer shell (VP7 and VP4) to become a double-layered particle (DLP) consisting of two inner-shell proteins VP2 and VP6 and the two core proteins. The DLP transcribes the genome using its own polymerase complex and the mature mRNA emerges along the fivefold axes. The trimeric VP6 has been associated with the viral transcriptase activity, as the removal of VP6 from the DLP yields core particles that are transcriptionally inactive. A range of MAbs against VP6 have been produced; some inhibit the transcriptase activity of the DLP (e.g. MAb-238), while others have no effect on transcription (e.g. MAb-133s; Kohli et al., 1994). By combining cryo-EM maps of the DLP-Fab complexes with the X-ray crystallographic atomic coordinates of the constituent molecules, we are gaining an insight into the
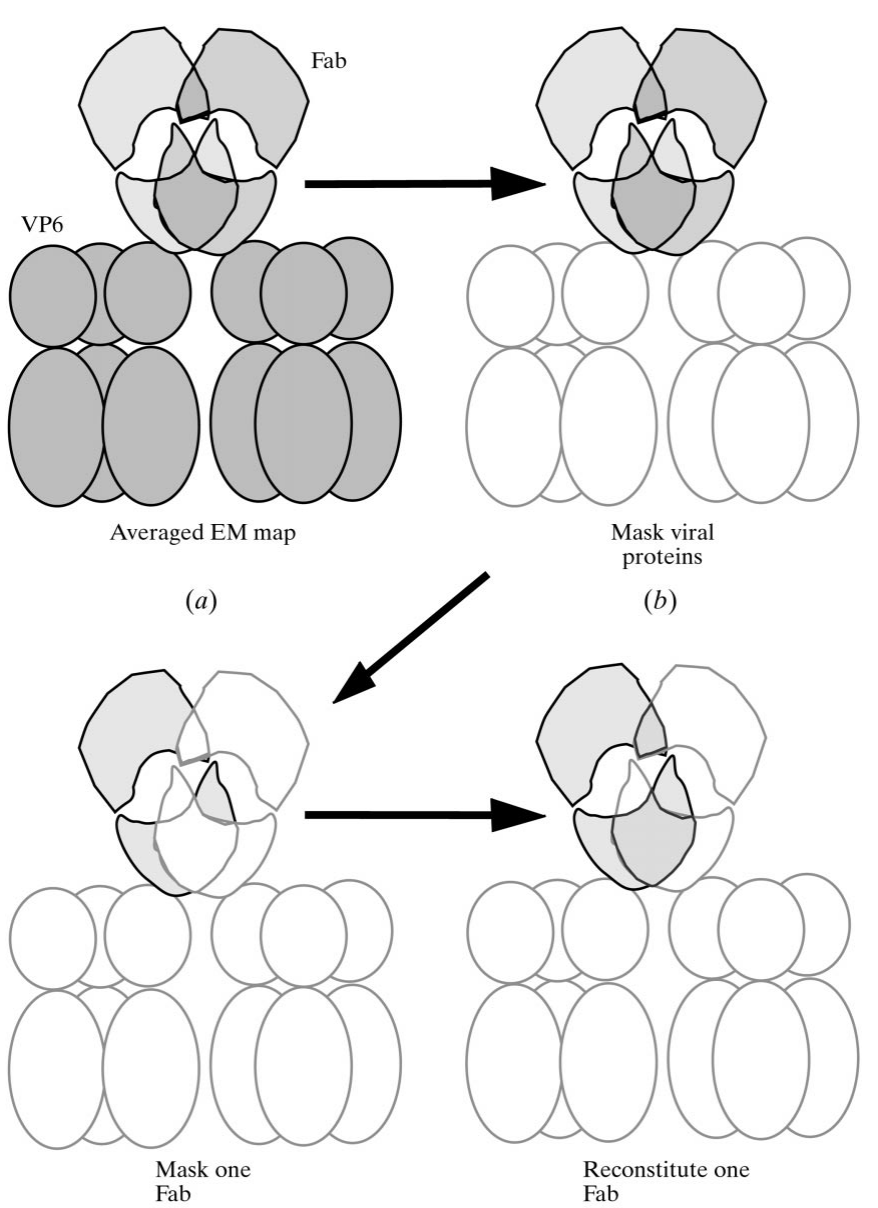

(c)

(d)

Figure 4

(a) Schematic diagram of one pair of overlapping Fabs bound to neighbouring VP6 trimers. Each VP6 trimer has three epitopes which do not overlap. The first step in both fitting methods is to mask off the density corresponding to the VP6 $(b)$. In the extraction method one Fab is masked off $(c)$ and in the overlap zone of the Fabs a proportion of the density is replaced in the map to recreate one Fab. 
mechanism by which certain MAbs inhibit the DLP transcriptase activity and the role played by VP6 in transcription (Thouvenin et al., in preparation). The three-dimensional reconstructions of these DLP-Fab complexes from cryo-EM shows that there is steric hindrance between the Fabs on neighbouring VP6 trimers (Fig. 4) in each case. So the maximum number of Fabs per DLP is only half the number of VP6 monomers, i.e. 780/2 $=390$. The ratio of Fabs on neighbouring sites ranges from 1:1 to 1:1.6.

We have applied our fitting programs on these maps of overlapping Fabs. Both fitting methods were satisfactory in

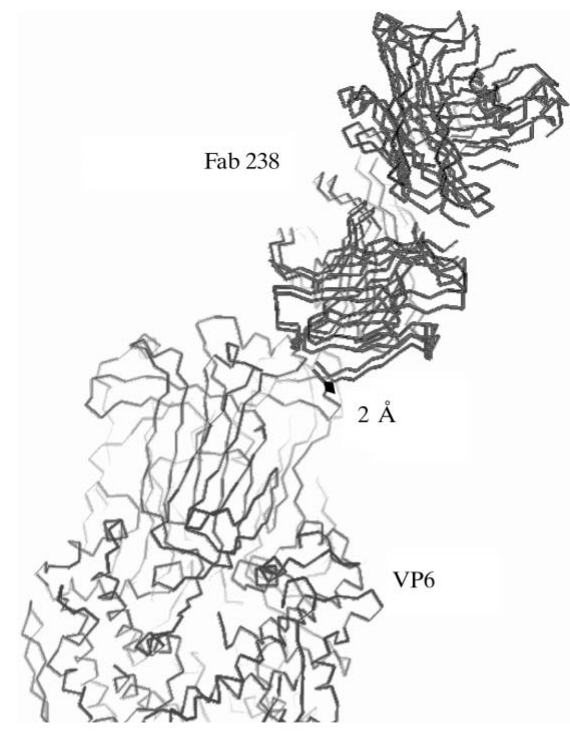

(a)

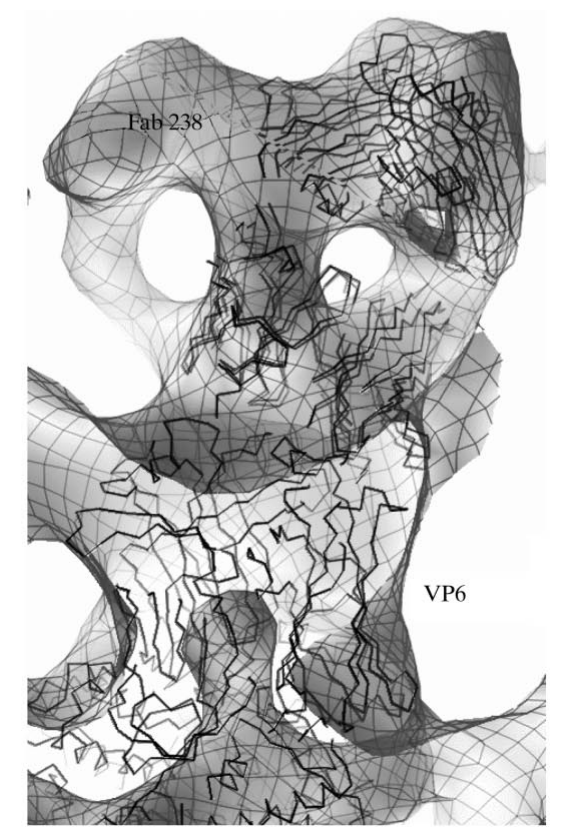

(c)

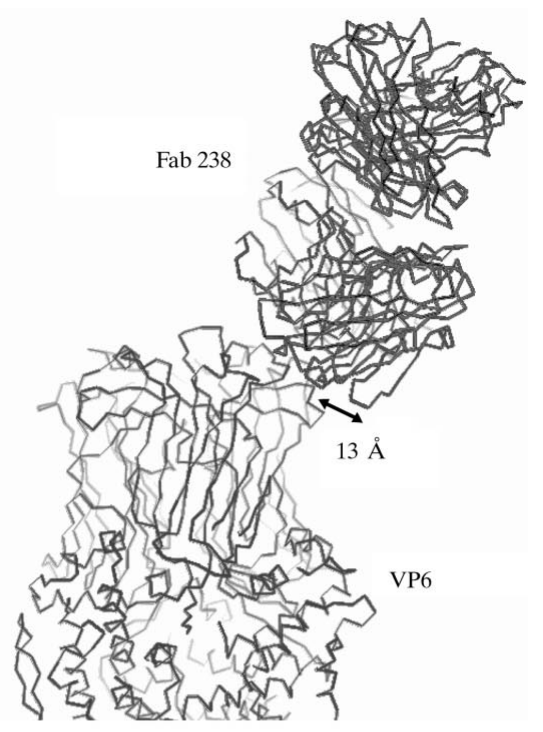

(b)

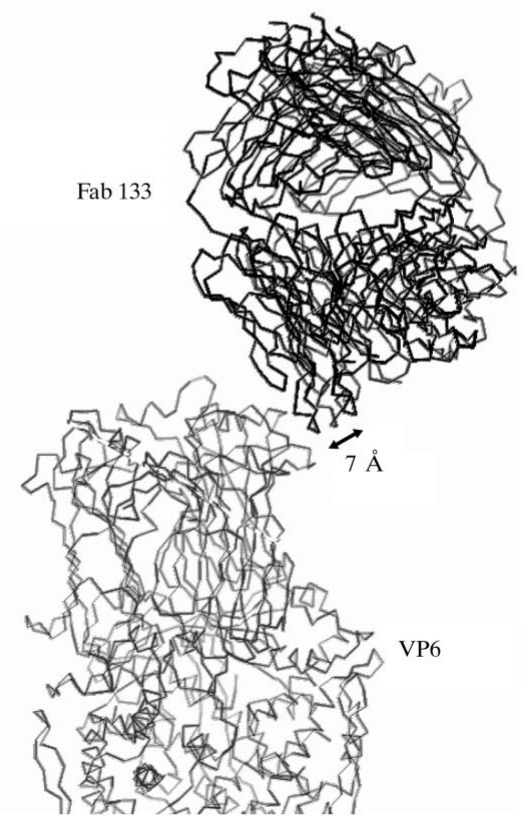

(d)
Figure 5

Fitting of VP6 and Fabs into the cryo-EM map of the DLP-Fab complexes. Comparison of the position of the fitted Fab-238 and position of the Fab in the atomic structure of the VP6-Fab complex for a Fab with an occupancy of $67 \%(a),(c)$ and $42 \%(b)$. In $(c)$ the isodensity surface of the EM map is also shown. In $(d)$ the fit of three Fab-133 on the three epitopes of one VP6 trimer are compared after being 'superposed' by a rotation of $120^{\circ}$ about the VP6 threefold axis. that they converged, but the second method (extraction method) converged more rapidly since it involved less computation. Neither method is very sensitive to the Fab occupancy. For model calculations, a variation of $20 \%$ in the occupancy changes the cross-correlation coefficient by $1 \%$ and does not change the fitted position significantly. Hence, in general we did not try to refine the occupancy in the fitting procedure.

As a reliability check for the fitting methods and parameters employed, we create a map from the fitted atomic positions of the overlapping Fabs and VP6 limited to $25 \AA$ A resolution. This map is then used as the map for fitting the crystallographic Fab structures. The method and parameters used are considered satisfactory if all atoms are placed to within less than $1 \AA$ of their original position.

It is particularly interesting that for the fit of the Fab in the DLP-Fab-238 map control of the Fab position is possible because the structure of the VP6-Fab-238 complex is known (Vaney et al., in preparation). While the position of the constant module of the Fab may vary, the position of the Fab paratope in contact with VP6 is assumed to remain invariant. The occupancy of the Fab-238s ranges between 40 and $71 \%$. There is an approximate inverse correlation between this occupancy and the error in the fitted position of the Fab paratope (Table 1). The Fabs with an occupancy of around $65 \%$ can be placed to within $2 \AA$ of the $\mathrm{X}$-ray position of the paratope, while those with an occupancy of around $40 \%$ stray by as much as $13 \AA$ (Figs. $5 a$ and $5 b$; Table 1).

Although the atomic structure of the VP6-Fab-133 complex is not known, it is still possible to estimate an error in the positioning of the Fab-133 paratope. By comparing the fitted positions of the three Fab-133s on one VP6 trimer rotated so they are superposed on one VP6 monomer, the spread in positions of the paratope gives an indication of the precision of fit of the Fab. The occupancies of the Fab-133s lie between 36 and 52\%. In the example shown in Fig. 5(d), the position of the paratope varies by less than $\pm 5 \AA$ around the mean position.

\section{Estimation of molecular occupancy from the density in EM maps}

It is tempting to suppose that the density in EM maps is correlated to the occupancy 
Table 1

Precision of fit as a function of occupancy for the extraction method.

The distances between the Fab-238 fitted into the EM map of the DLP-Fab238 complex and the Fab in the atomic structure of the VP6-Fab-236 complex are listed for a selection of Fabs. The distance indicated is for the $\mathrm{C}_{\alpha}$ of the residue Asn54, which is on the paratope of the Fab.

\begin{tabular}{ll}
\hline $\begin{array}{l}\text { Fab occupancy } \\
(\%)\end{array}$ & $\begin{array}{l}\text { Distance between fitted } \\
\text { and X-ray position }(\AA)\end{array}$ \\
\hline 67 & 1.7 \\
66 & 0.9 \\
58 & 5.2 \\
56 & 4.9 \\
44 & 16.2 \\
42 & 12.8 \\
\hline
\end{tabular}

and mobility of the corresponding molecule (Stewart et al., 1997; Chiu et al., 1999). However, quantifying this relation is not without error at the resolution of $20-30 \AA$ considered here. We have tried several different methods of evaluating the occupancy. They involve the comparison of the maximum density in each molecule or comparison of the average density in the volume of each molecule or part of the molecule.

It is revealing to look first at the density in each of the VP6 trimers in the DLP reconstruction at $19 \AA$ resolution. There is a variation of $\pm 15 \%$ in the maximum density in these trimers, even though we suppose that each trimer position has $100 \%$ occupancy. There is an even greater variation in the maximum density of the inner and outer domains of the VP6 trimer, where we might be even more certain that both domains are occupied to the same extent. It is thus clear that the partial occupancies of Fabs which we determine cannot be more precise than this.

Evaluation of the ratio of the maximum density for the constant module in each Fab and the maximum density in the map gives a reasonable first approximation for the occupancies. The sum of the occupancy of each pair of overlapping Fabs lies between 80 and $100 \%$ for the DLP-Fab-238 complex and between 60 and $75 \%$ for the DLP-Fab- 133 complex. Thus, as expected, the sum of occupancies is less than or equal to $100 \%$ and it is apparent that the overall Fab occupancy in the DLP-Fab-238 complex is close to maximal and in the DLPFab-133 complex the occupancy is roughly $65 \%$ of the maximum. If instead of the maximum density value the average maximum value in the VP6 trimers is used as reference, the sum of occupancies of some of the pairs of overlapping Fabs is greater than $100 \%$ (Table 2). Given the variation of $\pm 15 \%$ of the VP6 occupancies, this is probably a more reasonable estimate. Similarly, comparison of the average density in the constant module of the Fabs with the average density in a VP6 trimer also leads to values which are higher in general.

\section{Conclusions}

In general, for EM maps with a resolution of 20-30 ̊ the precision of fit of an atomic model is estimated to be close to $4 \AA$. This is true when the molecular occupancy is high, typi-
Table 2

Occupancies for each pair of overlapping Fabs in the DLP-Fab-238 and the DLP-Fab-133 complexes.

Occupancies were estimated from the maximum value in each Fab compared with the average maximum value in the VP6.

\begin{tabular}{llrlll}
\hline & DLP-Fab-238 & & & DLP-Fab-133 \\
\cline { 2 - 3 } Pairs of Fab & Occupancy $(\%)$ & Total & & Occupancy $(\%)$ & Total \\
\hline Fivefold & 67,42 & 109 & & 38,40 & 78 \\
Superposed & 97 & 97 & & 66 & 66 \\
Twofold & 57,57 & 114 & & 36,36 & 72 \\
& 56,44 & 100 & & 52,45 & 97 \\
& 66,58 & 124 & & 43,48 & 91 \\
& 71,44 & 115 & & 39,47 & 86 \\
& 63,40 & 103 & 38,49 & 87 \\
\hline
\end{tabular}

cally close to $100 \%$. We have found that this estimated precision remains true while the occupancy is above $50 \%$, but when the occupancy falls below this value the precision falls off rapidly. As judged by eye, the programs position the Fabs into the lower density quite well. The problem appears to be that the density attributed to molecules with low occupancy in the EM map is not perfectly reliable at this resolution. This could be a consequence of CTF effects in combination with the partial occupancy. The calculated map is an averaged map with contributions from the images of complexes each with a different pattern of bound Fabs. The extent of such effects will depend on the geometry of binding. For example, for Fab-133, where the variable modules of neighbouring Fabs overlap almost exactly, these effects are apparently less important than for Fab-238, where there is less overlap. The precision of estimated occupancies under similar conditions is limited by variations in the EM density map which do not correspond to real variations in the occupancy. Since different copies of the same molecule supposed to be present with $100 \%$ occupancy show a variation in density of roughly $\pm 15 \%$, the occupancy of molecules present at lower occupancy cannot be estimated with greater precision. It is clear from recent CTF-corrected cryo-EM maps at higher (7-15 $\mathrm{\AA}$ ) resolution (Conway et al., 1998) that the density in each molecule is more uniform, so there is reason to suppose that the limitations we encountered at $25 \AA$, both in precision of fit and estimation of occupancy. will be less severe at $10 \AA$.

\section{References}

Baker, T. S. \& Johnson, J. E. (1996). Curr. Opin. Struct. Biol. 6, 585594.

Belnap, D. M., Kumar, A., Folk, J. T., Smith, T. J. \& Baker, T. S. (1999). J. Struct. Biol. 125, 166-175.

Che, Z. W., Olson, N. H., Leippe, D., Lee, W. M., Mosser, A. G., Rueckert, R. R., Baker, T. S. \& Smith, T. J. (1998). J. Virol. 72, 4610-4622.

Chiu, C. Y., Mathias, P., Nemerow, G. R. \& Stewart, P. L. (1999). J. Virol. 73, 6759-6768.

Collaborative Computational Project, Number 4 (1994). Acta Cryst. D50, 760-763.

Conway, J. F., Cheng, N., Zlotnick, A., Stahl, S. J., Wingfield, P. T., Belnap, D. M., Kanngiesser, U., Noah, M. \& Steven, A. C. (1998). J. Mol. Biol. 279, 1111-1121. 
Conway, J. F. \& Steven, A. C. (1999). J. Struct. Biol. 128, 106-118.

Hewat, E. A. \& Blaas, D. (1996). EMBO J. 15, 1515-1523.

Hewat, E. A., Marlovits, T. C. \& Blaas, D. (1998). J. Virol. 72, 43964402.

Hewat, E. A., Verdaguer, N., Fita, I., Blakemore, W., Brookes, S., King, A., Newman, J., Domingo, E., Mateu, M. G. \& Stuart, D. I. (1997). EMBO J. 16, 1492-1500.

Jones, T. A., Zou, J. Y., Cowan, S. W. \& Kjeldgaard, M. (1991). Acta Cryst. A47, 110-109.

Kohli, E., Pothier, P., Tosser, G., Cohen, J., Sandino, A. M. \& Spencer, E. (1994). Arch. Virol. 135, 193-200.

Lawton, J. A., Estes, M. K. \& Prasad, B. V. (1999). Proc. Natl Acad. Sci. USA, 96, 5428-5433.

Mosser, A. G., Leippe, D. M. \& Rueckert, R. R. (1989). Molecular Aspects of Picornavirus Infection and Detection, edited by $\mathrm{B}$. Semler \& E. Ehrenfeld, pp. 155-167. Washington DC: American Society for Microbiology.

Porta, C., Wang, G., Cheng, H., Chen, Z., Baker, T. S. \& Johnson, J. E. (1994). Virology, 204, 777-788.

Prasad, B. V., Hardy, M. E., Dokland, T., Bella, J., Rossmann, M. G. \& Estes, M. K. (1999). Science, 286, 287-290.
Prasad, B. V. V., Matson, D. O. \& Smith, A. W. (1994). J. Mol. Biol. 240, 256-264.

Smith, T. J., Chase, E. S., Schmidt, T. J., Olson, N. H. \& Baker, T. S. (1996). Nature (London), 383, 350-354.

Smith, T. J., Olson, N. H., Cheng, R. H., Chase, E. S. \& Baker, T. S. (1993). Proc. Natl Acad. Sci. USA, 90, 7015-7018.

Smith, T. J., Olson, N. H., Cheng, R. H., Liu, H., Chase, E. S., Lee, W. M., Leippe, D. M., Mosser, A. G., Rueckert, R. R. \& Baker, T. S. (1993). J. Virol. 67, 1148-1158.

Stewart, P. L., Chiu, C. Y., Huang, S., Muir, T., Zhao, Y., Chait, B., Mathias, P. \& Nemerow, G. R. (1997). EMBO J. 16, 1189-1198.

Thouvenin, E., Laurent, S., Madelaine, M. F., Rasschaert, D., Vautherot, J. F. \& Hewat, E. A. (1997). J. Mol. Biol. 270, 238-246.

Tormo, J., Blaas, D., Parry, N. R., Rowlands, D., Stuart, D. \& Fita, I. (1994). EMBO J. 13, 2247-2256.

Trus, B. L., Newcomb, W. W., Booy, F. P., Brown, J. C. \& Steven, A. C. (1992). Proc. Natl Acad. Sci. USA, 89, 11508-11512.

Verdaguer, N., Schoehn, G., Ochoa, W. F., Fita, I., Brookes, S., King, A., Domingo, E., Mateu, M. G., Stuart, D. \& Hewat, E. A. (1999). Virology, 255, 260-268.

Wang, G. J., Porta, C., Chen, Z. G., Baker, T. S. \& Johnson, J. E. (1992). Nature (London), 355, 275-278. 